\title{
Accuracy and Practicality of a NIRS Device on Blood Lactate Levels
}

\author{
John Abbott, Melissa A. Whidden. West Chester University, West Chester, PA
}

Historically, cyclists have relied upon blood lactate analysis and/or metabolic testing to determine their fitness level and training zones. However, these methods are now being challenged by Near Infrared Spectroscopy (NIRS). The BSX Insight is a wearable NIRS device that claims to predict lactate threshold by analyzing changes in muscle oxygenation.

PURPOSE: The purpose of this study was to compare the accuracy of the BSX Insight against blood lactate and ventilatory threshold analyses. METHODS: Nine highly trained cyclists (1855 years of age) performed a graded exercise test while wearing the Insight and data was simultaneously collected via the TrueOne 2400 metabolic cart and Lactate Plus analyzer. Lactate threshold heart rate (HR) and power as predicted by the Insight was compared with blood lactate threshold (LT) as determined by the Dmax method and with the ventilatory threshold (VT) as determined by the respiratory exchange ratio (RER) method,V-slope method, and ventilatory equivalency (VEQ) method. RESULTS: Average LT power output as determined by the BSX Insight was not significantly different when compared to Dmax, RER, VEQ, and Vslope $(\mathrm{P}=0.811)$. Average LT power output from the Insight showed a positive correlation with Dmax $(r=0.869)$, RER $(r=0.964)$, VEQ $(r=0.970)$, and Vslope $(r=0.949)$. Average LT HR as determined via the BSX Insight device was not statistically different when compared to Dmax, RER, VEQ, and Vslope $(\mathrm{P}=0.386)$. The average percent difference of LT HR from the Insight compared with all other methods was within 5\% (Dmax $=2.29 \%$, RER $=$ $-2.56 \%, \mathrm{VEQ}=-2.74 \%$, Vslope $=0.545$ ). CONCLUSIONS: The wearable BSX Insight device is good at predicting LT HR and power output during a cycling test and muscle oxygenation can be used to estimate LT. The BSX Insight device may be an extremely helpful tool for those cyclists who do not have the ability or access to utilize more expensive testing protocols.

Supported by the College of Health Sciences Student-Faculty Research Award 